Portland State University

PDXScholar

\title{
Analytically Continued Hypergeometric Expression of the Incomplete Beta Function
}

Jack C. Straton

Portland State University, straton@pdx.edu

Follow this and additional works at: https://pdxscholar.library.pdx.edu/phy_fac

Part of the Mathematics Commons, and the Physics Commons

Let us know how access to this document benefits you.

\section{Citation Details}

Straton, Jack C., "Analytically Continued Hypergeometric Expression of the Incomplete Beta Function" (2002). Physics Faculty Publications and Presentations. 248.

https://pdxscholar.library.pdx.edu/phy_fac/248

This Post-Print is brought to you for free and open access. It has been accepted for inclusion in Physics Faculty Publications and Presentations by an authorized administrator of PDXScholar. Please contact us if we can make this document more accessible: pdxscholar@pdx.edu. 


\title{
Analytically Continued Hypergeometric Expression of the Incomplete Beta Function
}

\author{
Jack C. Straton \\ [This is the accepted version of an article appearing in Results in Mathematics \\ May 2002, Volume 41, Issue 3, pp 394-395, DOI 10.1007/BF03322781.]
}

\begin{abstract}
The Incomplete Beta Function is rewritten as a Hypergeometric Function that is the analytic continuation of the conventional form, a generalization of the finite series, which simpifies the Stieltjes transform of powers of a monomial divided by powers of a binomial.
\end{abstract}

1991 Mathematics Subject Classification: 33B20, 33C05, 44A15

Key Words: Incomplete beta function, hypergeometric function, Stieltjes transforms, definite integrals

The finite hypergeometric series expression for the Incomplete Beta Function, [1]

$$
{ }_{2} F_{1}(-n, 1 ; c ; z)=(1-c) z^{1-c}(z-1)^{n+c-1} B_{1-1 / z}(1-c-n, n+1),
$$

may be generalized to

\section{Theorem}

$$
\begin{aligned}
{ }_{2} F_{1}(-\nu, 1 ; \gamma ; z)=(1-\gamma) z^{1-\gamma}(z-1)^{\nu+\gamma-1} & {\left[B_{1-1 / z}(1-\gamma-\nu, \nu+1)\right.} \\
- & \left.B(1-\gamma-\nu, \nu+1)\left(1-\frac{(-1)^{-\nu} \sin [\pi(\gamma+\nu)]}{\sin (\pi \gamma)}\right)\right] .
\end{aligned}
$$

The Incomplete Beta Function [2] is conventionally defined [3] with real parameters for statistical problems,

$$
B_{x}(p, q)=\int_{0}^{x} t^{p-1}(1-t)^{q-1} d t \quad(0 \leq x \leq 1, \quad p, q>0),
$$

but is a smooth function of $p, q$ or $x$ when any or all are taken off the real axis (though it diverges as $x$ takes on large, real values). Its hypergeometric expression [4] is likewise well-behaved for complex parameters, so we rewrite this expression in its more general form

$$
\begin{aligned}
{ }_{2} F_{1}(\alpha, \beta ; \beta+1 ; w) & =\beta w^{-\beta} B_{w}(\beta, 1-\alpha)=\beta w^{-\beta} B(\beta, 1-\alpha)\left(1-I_{1-w}(1-\alpha, \beta)\right) \\
& =\beta w^{-\beta}\left[B(1-\alpha, \beta)-B_{1-w}(1-\alpha, \beta)\right] .
\end{aligned}
$$

One may analytically continue the left-hand side to [5]

$$
\begin{aligned}
{ }_{2} F_{1}(\alpha, \beta ; \beta+1 ; w) & =(-1)^{-\alpha}(w)^{-\alpha} \frac{\Gamma(\beta+1) \Gamma(\beta-\alpha)}{\Gamma(\beta) \Gamma(\beta+1-\alpha)}{ }_{2} F_{1}(\alpha, \alpha-\beta ; \alpha+1-\beta ; 1 / w) \\
& +(-1)^{-\beta}(w)^{-\beta} \frac{\Gamma(\beta+1) \Gamma(\alpha-\beta)}{\Gamma(\alpha) \Gamma(1)}{ }_{2} F_{1}(\beta, 0 ; \beta+1-\alpha ; 1 / w),
\end{aligned}
$$

Then equating right-hand sides of (4) and (5) and transforming the nontrivial hypergeometric function again [6] gives 


$$
\begin{aligned}
\left(B(1-\alpha, \beta)-B_{1-w}(1-\alpha, \beta)\right) & =(-1)^{-\alpha} w^{-\alpha+\beta} \frac{1}{(\beta-\alpha)}\left(1-\frac{1}{w}\right)^{1-\alpha}{ }_{2} F_{1}(1-\beta, 1 ; \alpha+1-\beta ; 1 / w) \\
& +(-1)^{-\beta} B(1-\alpha, \beta) \frac{\Gamma[1-(\alpha-\beta)] \Gamma(\alpha-\beta)}{\Gamma(1-\alpha) \Gamma(\alpha)}
\end{aligned}
$$

Letting $z=1 / w$ this simplifies [7] to

$$
\begin{aligned}
B_{1-1 / z}(1-\alpha, \beta) & =z^{\alpha-\beta} \frac{1}{(\beta-\alpha)}(z-1)^{1-\alpha}{ }_{2} F_{1}(1-\beta, 1 ; \alpha+1-\beta ; z) \\
& +B(1-\alpha, \beta)\left(1+(-1)^{1-\beta} \frac{\sin [\pi \alpha)]}{\sin [\pi(\alpha-\beta)]}\right),
\end{aligned}
$$

Finally one substitutes $\beta=\nu+1$ and $\alpha=\gamma+\beta-1$ and rearranges sides to obtain Eq. (2).

In addition, if one substitutes $\beta=1-\nu, \alpha=2-\mu$, and $z=\frac{\beta}{\gamma}$ and analytically continues the Gauss function, [8] one may obtain a more useful form for the known [9] Stieltjes transform [10] of powers of a monomial divided by powers of a binomial,

Corollary

$$
\begin{aligned}
\int_{0}^{\infty} \frac{x^{\nu-1}(\beta+x)^{1-\mu}}{\gamma+x} d x & =2 \int_{0}^{\infty} \frac{x^{\nu-1 / 2}\left(\beta+x^{2}\right)^{1-\mu}}{\gamma+x^{2}} d x=\pi \gamma^{\nu-1}(\beta-\gamma)^{1-\mu} \csc (\nu \pi) I_{1-\frac{\gamma}{\beta}}(\mu-1,1-\nu) \\
& =\pi \gamma^{\nu-1}(\beta-\gamma)^{1-\mu} \csc (\nu \pi)\left(1+(-1)^{\nu} \frac{\sin [\pi(2-\mu)]}{\sin [\pi(1+\nu-\mu)]}\right) \\
& -\frac{\pi \csc (\nu \pi) \beta^{\nu+1-\mu}}{(\mu-1-\nu)(\beta-\gamma) B(\mu-1,1-\nu)}{ }_{2} F_{1}\left(2-\mu, 1 ; 2-\mu+\nu ; \frac{\beta}{\beta-\gamma}\right),
\end{aligned}
$$

$(|\arg \gamma|<\pi|,| \arg \beta|<\pi|, 0<\operatorname{Re} \nu<\operatorname{Re} \mu)$ which is a finite series for integer $\mu>1$.

[1] A.P. Prudnikov, Yu. A. Brychkov and O.I. Marichev, Integrals and Series (Gorden and Breach, New York, 1990) v3, p. 466, No. 7.3.1.177.

[2] Handbook of mathematical functions with formulas, graphs, and mathematical tables, ed M. Abramowitz and I. A. Stegun(National Bureau of Standards, Applied mathematics series, 55, Washington, 1970), p. 263, Nos. 6.6.8, 6.6.2, and 6.6.3; see also K. Pearson, Biometrika 16, 202 (1924); M. J. Tretter and G. W. Walster, SIAM J. Sci. Stat. Comput. 1, 321 (1980); N. M. Temme, SIAM J. Math Anal. 18, 1638 (1987); B. W. Brown and L. B. Levy, ACM Trans. Math. Software 20, 393 (1994); and B. G. S. Doman, Math. Comput. 65, 1283 (1996).

[3] Larry C. Andrews, Special Functions for Engineers and Applied Mathematics, (MacMillan, New York, 1985) p. 70.

[4] I.S. Gradshteyn and I.M. Ryzhik, Table of Integrals, Series, and Products (Academic, New York, 1980), p. 950, No. 8.391.

[5] ibid., p. 1043, No. 9.132.2.

[6] ibid., p. 1043, No. 9.131c.

[7] ibid., p. 937, No. 8334.3.

[8] ibid., p. 1043, No. 9.131b.

[9] ibid., p. 290, No. 3.227.2; A. Erdélyi et al., Tables of Integral Transforms, Vol. II (McGraw Hill, New York, 1954), p. 217, No. 10.

[10] See, for instance, D. B. Sumner, Bull. Am. Math. Soc. 55, 174 (1949); A. Byrne and E. R. Love J. Austral. Math. Soc. 18, 328 (1974) and J. Math. Anal. \& Appl. 190, 428 (1995).

Jack C. Straton

University Studies

Portland State University

Portland, OR, 97207-0751

USA

Eingegangen am 10. November 2000 